\title{
Avalanche and spreading exponents in systems with absorbing states
}

\author{
Miguel A. Muñoz ${ }^{1,2}$, Ronald Dickman ${ }^{3}$, Alessandro Vespignani ${ }^{1}$, and Stefano Zapperi ${ }^{4}$ \\ 1 The Abdus Salam International Centre for Theoretical Physics (ICTP) P.O. Box 586, 34100 Trieste, Italy \\ 2 Dipartimento di Fisica e unità INFM, Universitá di Roma "La Sapienza", Piazzale A. Moro 2, I-00185 Roma, Italy \\ ${ }^{3}$ Departamento de Física, Universidade Federal de Santa Catarina, Campus Universitário \\ Trindade, CEP 88040-900, Florianópolis - SC, Brazil \\ ${ }^{4}$ PMMH-ESPCI, 10, rue Vauquelin, 75231 Paris cedex 05, France
}

(November 25, 2021)

We present generic scaling laws relating spreading critical exponents and avalanche exponents (in the sense of selforganized criticality) in general systems with absorbing states. Using these scaling laws we present a collection of the stateof-the-art exponents for directed percolation, dynamical percolation and other universality classes. This collection of results should help to elucidate the connections of self-organized criticality and systems with absorbing states. In particular, some non-universality in avalanche exponents is predicted for systems with many absorbing states.

PACS numbers: 64.60.Lx, 05.40.+j, 05.70.Ln

Directed percolation (DP) is broadly recognized as the paradigmatic example of systems exhibiting a transition from an active to an absorbing phase [1,2]. DP critical behavior appears in a vast array of systems, among others chemical reaction-diffusion models of catalysis [3], the contact process [ 国, damage spreading transitions [5], pinning of driven interfaces in random media [6], roughening transitions in one-dimensional systems [7], and Reggeon field theory [8]. This universality class has proven very robust with respect to the introduction of microscopic changes, and many apparently different systems share the same critical "epidemic" or "spreading" [9] and "bulk" exponents [1,2]. Nevertheless, examples of a system exhibiting a transition to an absorbing state outside the DP class have been identified in recent years. Some examples are:

- Systems with two symmetric absorbing states or, what is equivalent in many cases, systems in which the parity of the number of particles is conserved [10, 11].

- Systems with an infinite number of absorbing states, which exhibit nonuniversal spreading exponents [12,13].

- Systems in which the dynamics is limited to the interface between active and absorbing regions. These are in the class of the exactly solvable voter model [14], and compact directed percolation [15].

- Some models of epidemics with immunization (no reinfection) [16]. These belong to the so-called $d y$ namic percolation class; the final set of immune sites at criticality is a percolation cluster.
Recently, connections between self-organized criticality (SOC) and systems with absorbing states have attracted much attention. For example, there has been a debate on whether the extremal Bak-Sneppen for punctuated evolution [17] and certain variants are related to DP [18]. It has also been argued that sandpile models [19] share a number of features with systems having many absorbing states [20], and certain self-organized forest-fire models are related to dynamical percolation 21.

In self-organized models the so called avalanche exponents are customarily determined. Surprisingly, in spite of their obvious similarities, the general connections between spreading and avalanche exponents have not, to the best of our knowledge, been given explicitly for general systems with absorbing states. Establishing the general scaling laws relating avalanche and spreading exponents in systems with absorbing states is the main goal of what follows. This will allow us to put together many different scaling relations and exponent values, presently quite dispersed, and sometimes difficult to find in the literature, and should facilitate progress in this field.

Let us first define in detail spreading and avalanche critical exponents. The most accurate determination of the critical point of systems with absorbing states comes from "epidemic" or "spreading" experiments [9]. In these, a small perturbation (localized activity) is created at the origin of an otherwise absorbing configuration, leading to a spread of activity. In spreading experiments, it is customary to measure the number of particles, averaged over all runs (including those that have reached the absorbing state) $N(t)$, the survival probability $P(t)$, and the mean-squared deviation from the origin $R^{2}(t)$. At criticality these magnitudes scale as

$$
N(t) \sim t^{\eta} ; \quad P(t) \sim t^{-\delta} ; \quad R^{2}(t) \sim t^{z}
$$

where $\eta, \delta$ and $z$ [22] are the spreading exponents.

Once the critical point has been located accurately all the remaining standard critical indices can be estimated. For reference we show in Table I a compilation of the state-of-the-art values for the usual exponents in directed percolation, corresponding to a synthesis of the best series expansion and simulation results. For the sake of completeness let us give here their respective definitions. Calling $\Delta$ the distance to the critical point in terms of the reduced control parameter, $\rho$ the order parameter, $\xi_{\perp}\left(\xi_{\|}\right)$the characteristic length (time), $h$ 
an external field coupled to $\rho$, and $\chi \equiv L^{d} \operatorname{var}(\rho)$ the static "susceptibility", we have: $\rho \sim \Delta^{\beta}, \rho \sim h^{1 / \delta_{h}}$ at the critical point, $\chi \sim \Delta^{-\gamma^{\prime}}, \xi_{\perp} \sim \Delta^{-\nu_{\perp}}, \xi_{\|} \sim \Delta^{-\nu_{\|}}$, $P_{\infty} \equiv \lim _{t \rightarrow \infty} P(t) \sim \Delta^{\beta^{\prime}}$, and $\rho(t) \sim t^{-\theta}$ at the critical point.

¿From the whole set of exponents that can be defined in DP, only three are independent; the rest can be determined using well-known scaling relations (see appendix). In certain systems possessing an infinite number of absorbing states 12,13, a fourth independent critical exponent has to be introduced [23,24]. This is due to the fact that the exponent $\delta$ (which in DP coincides with $\theta$ ) is non-universal and depends on the nature of the absorbing state in which the epidemic spreads 23,24. (Similarly, the exponent $\beta^{\prime}$, normally identical to $\beta$, varies along with $\delta$ in such systems 23.)

On the other hand, studies of avalanche transport employ a different definition of the spread of activity. For instance, in the prototypical sandpile model [19] avalanches are obtained by adding one sand grain to a stable or absorbing configuration. In this way the system jumps among absorbing configurations via avalanche-like rearrangements. The following quantities and associated exponents are usually measured:

$$
\begin{aligned}
P(s) & \sim s^{-\tau} f\left(s / s_{c}\right) \\
s_{c} & \sim \epsilon^{-1 / \sigma} \\
\langle s\rangle & \sim \epsilon^{-\gamma}
\end{aligned}
$$

where $s$ is the size of an avalanche, (total number of active or toppling sites), $P(s)$ the associated probability distribution, $s_{c}$ the cut-off size, $\langle s\rangle$ the mean size, and $\epsilon$ represents the temperature-like variable associated with the process: $\Delta$ for contact process or DP, the dissipation rate for sandpiles, $F-F_{c}$ in driven-interface models (here $F$ is the driving force). If $\epsilon=0$, the characteristic length is defined by the system size $L$ through the scaling relation $s_{c} \sim L^{D}$. Analogously, the following exponents associated with the duration $t$ are also measured

$$
\begin{aligned}
P(t) & \sim t^{-\tau_{t}} g\left(t / t_{c}\right) \\
t_{c} & \sim \epsilon^{-1 / \sigma_{t}} \\
\langle t\rangle & \sim \epsilon^{-\gamma_{t}} .
\end{aligned}
$$

Let us now provide the general scaling laws relating avalanche and spreading exponents in systems with absorbing states.

¿From the definitions of $\eta$ and $\delta$, it is evident that the total number of particles in surviving runs goes like $N_{s} \sim t^{\eta+\delta}$, and therefore its time integral is governed by the exponent $1+\eta+\delta$. Thus an avalanche that dies at time $t$ has a typical size $s \sim t^{1+\eta+\delta}$. The probability to die between times $t$ and $t+d t$ scales as $D(t) \sim t^{-\delta-1} d t$. Observe that the time is defined in such a way that after a 'toppling' (updating of a given site), it is increased by $\Delta t=1 / N_{s}(t)$ 25. Therefore the number of topplings per unit time is $N_{s}(t)$. To express $\tau, \sigma$ and $\gamma$ as functions of the spreading exponents, let us consider a specific avalanche size, say $s_{1}$. An avalanche of size $s_{1}$ can have different durations, since $t$ and $s$ are not related in a deterministic way, i.e.,

$$
P\left(s_{1}\right)=\int_{t_{1}}^{t_{2}} d t P\left(s_{1} \mid t\right) D(t),
$$

where $t_{1}$ and $t_{2}$ are the minimum and maximum times compatible with $s_{1}$, and $P(s \mid t)$ is the conditional probability of an avalanche having size $s$, given it dies at time $t$. $P(s \mid t)$ is bell-shaped, with its maximum at $t \sim s^{1 /(1+\eta+\delta)}$ 26]. Writing $P(s \mid t)=t^{-(1+\eta+\delta)} F\left(s / t^{1+\eta+\delta}\right)$, where $F(u)$, the (normalized) scaling function, is nonsingular, we have, on changing variables

$$
P(s)=s^{-(1+\eta+2 \delta) /(1+\eta+\delta)} \int d u u^{\delta /(1+\eta+\delta)} F(u)
$$

in other words,

$$
\tau=\frac{1+\eta+2 \delta}{1+\eta+\delta}
$$

With $t_{c} \sim \epsilon^{-\nu_{\|}}$, and using $s_{c} \sim t_{c}^{1+\eta+\delta}$, we have

$$
1 / \sigma=\nu_{\|}(1+\eta+\delta) \text {. }
$$

The remaining exponent $\gamma$ and the fractal dimension $D$ can be determined using the relations $\gamma=(2-\tau) / \sigma=$ $\nu_{\|}(1+\eta)$ (this last equation for $\gamma$ has already been found by other authors [27), and the standard relation $D=$ $1 /\left(\sigma \nu_{\perp}\right)$. Following a very similar derivation to the one just presented, one can easily determine also the following scaling relations for the exponents associated with $P(t)$ :

$$
\begin{aligned}
\tau_{t} & =1+\delta \\
\sigma_{t} & =\sigma(1+\eta+\delta)=1 / \nu_{\|} \\
\gamma_{t} & =\left(2-\tau_{t}\right) / \sigma_{t}=\nu_{\|}(1-\delta)
\end{aligned}
$$

Let us derive explicitly the expression for $\gamma_{t}$. In DP, we have, for $\Delta<0$, or for $\Delta=0$ and finite $L$, the scaling form for the survival probability $P(t) \sim t^{-\delta} e^{-t / t_{c}}$ with $t_{c} \sim|\Delta|^{-\nu_{||}}$, or $t_{c} \sim L^{\nu_{||} / \nu_{\perp}}$. Since the probability density for dying at time $t$ is $-d P(t) / d t$, we can write

$$
\begin{aligned}
\langle t\rangle=\int t \frac{d}{d t}[-P(t)] d t & \sim \int_{t_{0}}^{\infty} t^{-\delta} e^{-t / t_{c}} d t \\
& \sim t_{c}^{1-\delta} \int_{u_{0}}^{\infty} u^{-\delta} e^{-u} d u .
\end{aligned}
$$

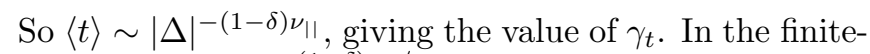
size case, $\langle t\rangle \sim L^{-(1-\delta) \nu_{||} / \nu_{\perp}}$ (observe that $t_{0}$ and $u_{0}$ are unimportant lower cut-offs).

All the scaling relations derived so far are general, and valid for all systems with absorbing states. Specific scaling relations for systems in the DP class can be written using the well known relation [9] $\eta+2 \delta=d z / 2$. Using the best values for the spreading exponents in DP taken from the bibliography (Table I), we obtain the values of 
the avalanche exponents for DP in different dimensions (they are also summarized in table I).

Applying our general relations to other classes of models, we obtain:

- For models with parity conservation, using the known result for spreading exponents [11, we predict $\tau \approx 1.22, \sigma \approx 0.24, \gamma \approx 3.25, \tau_{t} \approx 1.28$, $\sigma_{t} \approx 0.31$ and $\gamma_{t} \approx 2.33$ in $d=1$ [28] and mean field values above that dimension. These results have also been derived and numerically tested in [29].

- In systems with many absorbing states, a generalized hyperscaling relation has to be introduced, due to the fact that in this case the exponents $\delta$ and $\eta$ are non-universal and therefore $\delta \neq \theta$ in general (on the other hand the combination $\eta+\delta$ retains its $\mathrm{DP}$ value). The generalized scaling law for these systems is 23.24] $\eta+\delta+\theta=d z / 2$. Applying our scaling laws we predict non-universal values of $\tau, \gamma$, $\tau_{t}$ and $\gamma_{t}$ for systems with many absorbing states; i.e. if experiments are performed on a fixed environment, the results depend upon the environment itself. Recently this kind of non-universality has been observed in the class of sandpile models with fixed energy [30].

- For models in the CDP class we have $\tau=4 / 3$, $\sigma=2 / 3, \gamma=1, \tau_{t}=3 / 2, \sigma_{t}=1$ and $\gamma_{t}=1 / 2$ in $d=1$ and mean field values in $d=2$ and above.

- For dynamical percolation we can take advantage of our scaling laws, using them the other way around, i.e., using the well known avalanche (cluster) exponents for standard percolation 31,37 permits us to determine the spreading exponents [24] with good accuracy. In table II, we report a collection of exponent values in $d=2,3$ and 6 spatial dimensions.

In summary, we have presented the general scaling relations that rule general systems with absorbing states, and present a collection of exponent values that can be useful as a reference. We believe that this coherent derivation and collection of otherwise scattered scaling laws and exponent values may facilitate progress in drawing connections and similarities in many systems which show absorbing states and avalanche behavior.

ACKNOWLEDGMENTS- This work has been partially supported by the European network, project number FMRXCT980183. We thank K. B. Lauritsen, H. Park and R.M. Ziff for useful comments and remarks.

After completion of this work we became aware of a recent paper by Lauritsen et al. 38, in which very similar scaling relations to the ones proposed here are derived for directed percolation in the presence of an absorbing wall. In particular, as in systems with many absorbing states they find in that case $\delta \neq \theta$. A direct consequence is that, as discussed here, some avalanche exponents do not take their corresponding DP values. Some other interesting scaling relations can be found in 39].

\section{APPENDIX: Scaling relations for DP}

Here we present a collection of scaling laws for the DP universality class [1, 2, 9].

$$
\begin{aligned}
\eta+\delta+\theta & =d z / 2 \\
\delta_{h} & =\left(\nu_{\|}+d \nu_{\perp}\right) / \beta-1 \\
\beta & =\beta^{\prime} \\
\delta & =\theta \\
\gamma^{\prime} & =\gamma-\nu_{\|}=d \nu_{\perp}-2 \beta \\
\beta & =\theta \nu_{\|} \\
\beta^{\prime} & =\delta \nu_{\|} \\
z & =2 \nu_{\perp} / \nu_{\|} \\
D & =1 /\left(\sigma \nu_{\perp}\right)=d+\left(\nu_{\|}-\beta\right) / \nu_{\perp} \\
\gamma & =(2-\tau) / \sigma=d \nu_{\perp}+\nu_{\|}-2 \beta .
\end{aligned}
$$

Observe that not all of these relations are independent.

[1] J. Marro and R. Dickman, Nonequilibrium Phase Transitions and Critical Phenomena, Cambridge University Press, (Cambridge, 1996). R. Dickman, in Nonequilibrium Statistical Mechanics in One Dimension, Ed. V. Privman. Cambridge University Press, 1997. G. Grinstein and M. A. Muñoz, in Fourth Granada Lectures in Computational Physics, Ed. P. Garrido and J. Marro, Springer (Berlin) 1997. Lecture Notes in Physics, 493, 223 (1997).

[2] Percolation Structures and Processes, edited by G. Deutscher, R. Zallen, and J. Adler, Annals of the Israel Physical Society, Vol. 5 (Adam Hilger, Bristol, 1983).

[3] R. M. Ziff, E. Gulari, and Y. Barshad, Phys. Rev. Lett. 56, 2553 (1986); I. Jensen, H.C. Fogedby and R. Dickman, Phys. Rev. 41, 3411 (1990).

[4] T.E. Harris, Ann. Phys. 2, 969 (1974).

[5] P. Grassberger, J. Stat. Phys. 79, 13 (1995).

[6] See A. L. Barabasi and H. E. Stanley, Fractal Concepts in Surface Growth (Cambridge University Press, Cambridge, 1995); A. L. Barabasi, G. Grinstein, and M.A. Muñoz, Phys. Rev. Lett. 76, 1481, (1996) and references therein.

[7] J. Kertész and D. E. Wolf, Phys. Rev. Lett. 62, 2571 (1989).

[8] P. Grassberger, Z. Phys. B 47, 365 (1982); H.K. Janssen, Z. Phys. B 42, 151 (1981).

[9] P. Grassberger and A. de la Torre, Ann. Phys. (N.Y.) 122, 373 (1979).

[10] M. H. Kim and H. Park, Phys. Rev. Lett. 73, 2579 (1994); H. Hinrichsen, Phys. Rev. E 55, 219 (1997). 
[11] I. Jensen, Phys. Rev. E 50, 3623 (1994). H. Takayasu and A. Yu. Tretyakov, Phys. Rev. Lett. 68, 3060 (1992). See also J. Cardy and U.C. Täuber, Phys. Rev. Lett. 77, 4780 (1996) and references therein.

[12] I. Jensen and R. Dickman, Phys. Rev. E 48, 1710 (1993); I. Jensen, Int. J. Mod. Phys. B 8, 3299 (1994); I. Jensen, Phys. Rev. Lett. 70, 1465 (1993).

[13] See, M.A. Muñoz, G. Grinstein, R. Dickman, and R. Livi, Phys. Rev. Lett. 76, 451 (1996); Physica D 103 485 (1997); M.A. Muñoz, G. Grinstein and R. Dickman, J. Stat. Phys. 91, 541 (1998); and references therein.

[14] T. M. Liggett, Interacting Particle Systems, SpringerVerlag, (New York, 1985).

[15] E. Domany and W. Kinzel, Phys. Rev Lett. 53, 311 (1984); J. W. Essam, J. Phys. A 22, 4927 (1989).

[16] J.L. Cardy and P. Grassberger, J. Phys. A 18, L267 (1985); H.K. Janssen, Z. Phys. B 58, 311 (1985).

[17] P. Bak and K. Sneppen, Phys. Rev. Lett. 71, 4083 (1993). For a review see M. Paczuski, S. Maslov and P. Bak, Phys. Rev. E 53, 414 (1996).

[18] M. Paczuski, S. Maslov and P. Bak, Europhys. Lett. 27, 97 (1994). B. Jovanovic, S. V. Buldyrev, S. Havlin, and H. E. Stanley, Phys. Rev. E 50, R2403 (1994). P. Grassberger, Phys. Lett. A 200, 277 (1995). D. Sornette and I. Dornic, Phys. Rev. E 54, 3334 (1996).

[19] P. Bak, C. Tang and K. Wiesenfeld, Phys. Rev. Lett. 59, 381 (1987); Phys. Rev. A 38, 364 (1988).

[20] R. Dickman, A. Vespignani and S. Zapperi, Phys. Rev. E 57, 5095 (1998). A. Vespignani, R. Dickman, M. A. Muñoz and S. Zapperi, Phys. Rev. Lett. 81, 5676, (1998).

[21] See for example, S. Clar, B. Drossel, F. Schwabl, Phys. Rev. Lett. 75, 2722 (1995).

[22] Observe that this is not the dynamic exponent $z_{d}$ defined usually as $t_{c} \sim \xi_{\perp}^{z_{d}}$. The two exponents are related by $z=2 / z_{d}$.

[23] J.F.F. Mendes, R. Dickman, M. Henkel and M.C. Marques, J. Phys. A 27, 3019 (1994).

[24] G. Grinstein, M. A. Muñoz and Y. Tu, Phys. Rev. E. 56, 5101 (1997).

[25] This is for sequential updating. For parallel processes time is increased in one unit after each updating.

[26] We have verified numerically this assumption for DP as well as for a stochastic sandpile model. When this assumption is not satisfied, inconsistent results can be obtained: A. Chessa, H.E.Stanley, A. Vespignani and S. Zapperi, Phys. Rev. E 59, R12 (1999).

[27] I. Jensen, J. Phys. A 29, 7013 (1996); see also J. L. Cardy, R. L. Sugar, J. Phys. A 13, L423 (1980).

[28] These exponents corresponds to the case in which the absorbing state can be reached. If is not accessible, the exponent $\delta$ is zero 11], and the exponents $\tau, \tau_{t}, \gamma$ and $\gamma_{t}$ are modified.

[29] K. B. Lauritsen, P. Fröjdh and M. Howard, Phys. Rev. Lett. 81, 2104 (1998).

[30] M. A. Muñoz, R. Dickman, A. Vespignani, and S. Zapperi, Preprint 1998.

[31] See P. Grassberger, Math. Biosci. 63, 157 (1982). P. Grassberger, J. Phys. A 18 , L215 (1985); S. Havlin and R. Nossal, J. Phys. A 17, L427 (1984). For recent references see P. Grassberger, cond-mat/9808095.
[32] J. Adler and J.A.M.S. Duarte, Phys. Rev. B 35, 7046 (1987).

[33] P. Grassberger and Y. Zhang, Physica A 224, 169 (1996).

[34] C.A. Voigt and R.M. Ziff, Phys. Rev. E 56, R6241 (1997).

[35] I. Jensen, Phys. Rev. A 29, R563 (1992).

[36] J. Adler et al., Phys. Rev. B 37, 7529 (1988).

[37] A. Bunde and S. Havlin, Fractals and disordered systems (eds. A. Bunde and S. Havlin), Springer Verlag, Heidelberg 1991.

[38] K. B. Lauritsen, K. Sneppen, M. Markosova and M. H. Jensen, Physica A 247, 1 (1997). See also P. Fröjdh, M. Howard and K. B. Lauritsen, J. Phys. A 31, 2311 (1998).

[39] S. Maslov, Phys. Rev. Lett. 74, 562 (1995). 


\begin{tabular}{|c|l|l|l|l|}
\hline \hline \hline exponent & $d=1$ & $d=2$ & $d=3$ & $d=4$ \\
\hline \hline$\beta=\beta^{\prime}$ & $0.27649(4)^{a}$ & $0.583(4)^{c}$ & $0.805(10)^{g}$ & 1 \\
$1 / \delta_{h}$ & $0.111(3)^{b}$ & $0.285(35)^{b}$ & $0.45(2)^{h}$ & $1 / 2$ \\
$\gamma^{\prime}$ & $0.54386(7)^{a}$ & 0.35 & 0.19 & 0 \\
$\nu_{\|}$ & $1.73383(3)^{a}$ & $1.295(6)^{d}$ & $1.105(5)^{g}$ & 1 \\
$\nu_{\perp}$ & $1.09684(1)^{a}$ & $0.733(4)^{e}$ & $0.581(5)$ & $1 / 2$ \\
$\delta=\theta$ & $0.15947(3)^{a}$ & $0.4505(10)^{f}$ & $0.730(4)^{g}$ & 1 \\
$\eta$ & $0.31368(4)^{a}$ & $0.2295(10)^{f}$ & $0.114(4)^{g}$ & 0 \\
$z$ & $1.26523(3)^{a}$ & $1.1325(10)^{f}$ & $1.052(3)^{g}$ & 1 \\
$\nu_{||} / \nu_{\perp}$ & $1.58074(4)$ & $1.766(2)$ & $1.901(5)$ & 2 \\
\hline \hline$\tau$ & 1.108 & 1.268 & 1.395 & $3 / 2$ \\
$\sigma$ & 0.391 & 0.459 & 0.490 & $1 / 2$ \\
$\gamma$ & 2.277 & 1.593 & 1.232 & 1 \\
$D_{f}$ & 2.328 & 2.968 & 3.507 & 4 \\
$\tau_{t}$ & 1.159 & 1.450 & 1.730 & 2 \\
$\sigma_{t}$ & 0.576 & 0.771 & 0.904 & 1 \\
$\gamma_{t}$ & 1.457 & 0.711 & 0.298 & 0 \\
\hline \hline
\end{tabular}

Critical exponents for Directed Percolation. Exponents calculated by using scaling relations contained in this paper are reported in the lower part. ${ }^{a}$ See 27$] ;{ }^{b} 32$; ${ }^{c}$ obtained using $\beta=\delta \nu_{\|} ;{ }^{d}$ [33]; ${ }^{e}$ obtained using $\nu_{\perp}=z \nu_{\|} / 2 ;{ }^{f}$ 34]; ${ }^{g}$ [35]; $h$ [36]. Where not reported uncertainties are in the last digit. For $d=4$ we report the exact mean field values.

\begin{tabular}{|c|c|c|c|}
\hline \hline exponent & $d=2$ & $d=3$ & $d=6$ \\
\hline \hline$\beta=\beta^{\prime}$ & $5 / 36$ & 0.417 & 1 \\
$\nu_{\|}$ & 1.506 & 1.169 & 1 \\
$\gamma$ & $43 / 18$ & 1.795 & 1 \\
$\nu_{\perp}$ & $4 / 3$ & 0.875 & $1 / 2$ \\
$\tau$ & $96 / 91$ & 1.188 & $3 / 2$ \\
$\sigma$ & $36 / 91$ & 0.452 & $1 / 2$ \\
$D_{f}$ & $91 / 48$ & 2.528 & 4 \\
\hline \hline$\tau_{t}$ & 1.092 & 1.356 & 2 \\
$\sigma_{t}$ & 0.664 & 0.855 & 1 \\
$\gamma_{t}$ & 1.367 & 0.752 & 0 \\
$\eta$ & 0.586 & 0.536 & 0 \\
$\delta=\theta$ & 0.092 & 0.356 & 1 \\
$z$ & 1.771 & 1.497 & 1 \\
\hline \hline
\end{tabular}

Critical exponents for dynamical percolation. Exponents calculated by using scaling relations contained in this paper are reported in the lower part. The rest of exponents values are from [37]. Where not reported uncertainties are in the last digit. For $d=2$, values expressed as fractions refer to exact results 37. For $d=6$ we report the exact mean field values. 